The Royal Society, with its firm and unbroken tradition of complete aloofness from political controversy, may still find it an important part of its function to keep watch and, if necessary, to stand without compromise for the right and the duty of science to seek the truth for its own sake, in complete freedom from any kind of extraneous influence. I hope, indeed, that there will never be need thus to invoke our tradition, in order to protect the freedom and the integrity of science from the enthusiasm and the advocacy of any of its friends.

\title{
PHYSICAL CONCEPTS OF THE MESON THEORY OF THE ATOMIC NUCLEUS
}

\author{
By Prof. W. Heitler \\ Dublin Institute for Advanced Studies
}

\begin{abstract}
A SYMPOSIUM was recently held at the Dublin Institute for Advanced Studies, at which the present state of the meson theory was discussed. The present article discusses some of the views expressed, so far as they may be of interest for the general reader. Most of the views put forward in this article-if not generally known - have been discussed in recent years between Dr. H. Fröhlich and myself.
\end{abstract}

There is at the present time a veritable jungle of literature on the meson theory of the nucleus. Quite apart from the sometimes very extensive calculations, there exist at present two or three different meson theories-they differ essentially in the value attributed to the spin of the mesonand each is claimed to have great advantages. This in itself may be sufficient to stress the very preliminary character of the theory. The meson theory is entirely based upon the principles of quantum mechanics and of special relativity. Yet it could scareely be expected that these principles will be sufficient to solve the problem of the elementary particles; something going far beyond relativistic quantum mechanics will be needed for this purpose. Nevertheless, the meson theory has already yielded a number of very valuable results and suggestions which can be considered as safe whatever the future development may be. We may perhaps compare the situation with that obtained by applying Lorentz's classical theory of the electron to an atom, treating the latter as a classical oscillator. The most striking feature, the existence of stationary states, cannot be understood in this way, but a number of other features, such as the absorption and scattering of light, can be understood quite well. Thus we must not expect that the present meson theory can be used to calculate exactly the binding energy of the deuteron, or that it can give any other quantitative results ; but we can expect to obtain a large number of qualitative results and order of magnitude relations between the fundamental properties of the elementary particles. These properties turn out to be largely independent of the particular form we choose for the theory and can be explained by using general arguments only. (This does not mean that the development of the formalism is superfluous. It has indeed its merits-and very important ones -but a discussion of this lies outside the scope of the present article.)

The meson theory originated from an ingenious idea put forward by Yukawa in 1935. In order to describe the short-range forces prevailing between a proton and neutron, Yukawa introduced a new kind of field, $\varphi$, which was thought to be analogous to, but different in nature from, the electromagnetic field. The short range of these forces requires a modification of the field equations so that the static part of the field is now described by a modified 'Poisson' equation:

$$
\nabla^{2} \varphi+\lambda^{2} \varphi=0
$$

with its singular solution, $\varphi=g e^{-\lambda r} / r$. The range of the forces is approximately $1 / \lambda$. It is an experimental fact that $1 / \lambda$ is of the same order of magnitude as the classical electron radius $e^{2} / m c^{2}$. $g$ is a new universal constant with the dimensions of a charge. It determines the strength of the nuclear field. We derive its value below.

From (1) we can go over to a wave equation describing waves in vacuo:

$$
\nabla^{2} \varphi-\frac{1}{c^{2}} \varphi+\lambda^{2} \varphi=0 .
$$

(2) can also be considered as a relativistic wave equation for a particle, but in contrast to the corresponding equation in Maxwell's theory, it describes the free motion of a particle with a finite rest mass, $\mu=\hbar / c \lambda$. Using the fact that $1 / \lambda \fallingdotseq e^{2} / m c^{2}$ ( $m$ is electron mass), we obtain for the new mass the order of magnitude :

$$
\frac{\mu}{m} \doteqdot \frac{\hbar c}{e^{2}}=137
$$

Moreover, it has long been concluded from the 
saturation of the nuclear forces and other facts that the forces between a proton and neutron are connected with an exchange of electric charge. If this is taken literally, it must mean that the electric charge is not necessarily concentrated at the position of the proton but can also be found between the two particles. In other words, the nuclear field $\varphi$ must itself carry electric charge. The free particles described by (2) are consequently charged particles, positive and negative, as will be seen below.

Three years after Yukawa had published these ideas, particles with these properties were discovered in cosmic radiation and named 'mesons'. The actual mass was found to be about $180 \mathrm{~m}$, in good agreement with (3). Secondly, the cosmic ray mesons are charged (positive and negative). The agreement of these two facts with the prediction from purely nuclear considerations is so striking that there can be no doubt about the basic correctness of Yukawa's ideas.

Next we have to determine the value of the new universal constant $g$. For this purpose we use two more experimental facts: (i) The size $a$ of the deuteron is little greater than the range of the forces $1 / \lambda$. This is by no means trivial. The size of a hydrogen atom is the Bohr radius, whereas the range of the Coulomb forces is infinite. (ii) It is known that in the ground state of the deuteron the kinetic energy is almost equal to the potential energy, leaving only a small fraction of the potential energy for the binding energy. According to the uncertainty relation, the kinetic energy is

$$
E_{\text {kin. }} \doteqdot \frac{\Delta p^{2}}{M} \fallingdotseq \frac{\hbar^{2}}{a^{2} M} \fallingdotseq \frac{\hbar^{2} \lambda^{2}}{M}
$$

The potential energy is approximately $E_{\text {pot. }} \doteqdot g^{2} \lambda$. Thus we find $g^{2} \fallingdotseq \hbar^{2} \lambda / M$, or

$$
\frac{\hbar c}{g^{2}} \doteqdot \frac{M}{\mu} \doteqdot 10
$$

This gives a value for $g$ of about 4 elementary charges $e$. The similarity of (4) and (3) is very striking. The two dimensionless universal constants $e^{2} / \hbar c$ and $g^{2} / \hbar c$ are both equal to the ratio of the masses of two elementary particles. Both relations are deduced from experimental facts, and so far we have no deeper insight into the meaning of these relations.

We have looked upon the field $\varphi$ from two different aspects. On one hand, we have considered it as a field of force, and on the other hand, as a Schrödinger wave function for the meson as a particle. The two aspects are entirely equivalent. While the Maxwell field is primarily a field of force and can only be looked upon as a wave function for light quanta in special cases, and on the other hand the Schrödinger wave function of an electron never acts as a field of force, we can consider the meson field $\varphi$ from whichever point of view is more convenient. Indeed, the production of the static field $g e^{-\lambda r} / r$ by a proton or neutron is often described in the particle picture as a "virtual emission and reabsorption" of a meson. This picture can often be used with advantage.

What is the precise meaning of this expression? We have already seen that the field $\varphi$ carries an electric charge. If we would carry out an experiment to find the charge at a distance $r$, say, from the proton (or neutron), we shall, indeed, with a certain probability, find a charged particle at that point. (The charge can, of course, only appear in the form of a particle, that is, in an integral multiple of e.) The word emission in the above expression has thus a very literary meaning. On the other hand, such an emission of a particle with a rest mass $\mu$ would be contrary to the law of con. servation of energy. While all other conservation laws (charge, momentum, angular momentum, spin, statistical) are fulfilled during this emission, the word 'virtual' means that, in contrast to a real emission, energy need not be conserved. If wo therefore carry out an experiment to find the charge at a distance $r$ from the proton, this experiment will only give a positive result if the necessary energy $\mu c^{2}$ is supplied by the measuring process itself.

This argument can be used to derive the extension of the nuclear field in a direct way without referring to a particular wave equation. The argument is due to Wick, but we give it here in a somewhat modified form. We measure the position of the meson at a distance $r$ with an accuracy $\Delta r$ of the order of magnitude of $r$ itself. The particle will then have an average momentum $\triangle p=\hbar / r$. This is connected with an uncertainty of the energy $\Delta E \doteqdot \frac{\Delta p^{2}}{\mu}$ (if $\Delta p$ is not much larger than $\mu c$ ). $\triangle \boldsymbol{E}$ is also the energy supplied by the measuring process. In order that the actual finding of the particle is not contradictory to the conservation of energy, $\Delta E$ must at least be equal to $\mu c^{2}$. Thus the experiment can only be successful if $\hbar^{2} / \mu r^{2} \geq \mu c^{2}$ or

$$
r \leq \hbar / \mu c \text {. }
$$

This expression gives the extension of the region in which the meson can be found, in other words, the extension of the nuclear field; and is identical with Yukawa's relation.

The next question is: How big is now the probability $\alpha$ of finding the meson outside at a distance $r$ from the proton? Here the limitations of the theory become apparent. $\alpha$ depends, of course, on $r$, but it is clear that $r$ must not be chosen too small. First of all, no meaning can be attached 
to a dissociation where the meson is practically at the position of the proton, since this state is indistinguishable from the undissociated state. If $r$ is smaller than $\hbar / M c$, the measuring process supplies energies larger than $M c^{2}$, and pairs of heavy particles can be created. The picture of a dissociated proton then fails completely. Even if we choose $r=\hbar / \mu c$, the concept of emission of a single meson fails, since then pairs of mesons can be created. To be on the safe side, we have therefore to restrict our concept to distances not much smaller than $1 / \lambda$ : But these are just the distances where the physical effects are of interest. Although for many problems the theory can actually be applied to much smaller distances, the simple concepts developed in this article break down in the very region in which they are of most interest. We cannot expect any quantitative results from them, but in the region of $\hbar / \mu c$ the results will be of qualitative significance.

To find the probability of dissociation, we calculate the total energy contained in the field outside $1 / \lambda$ and divide it by the energy of the meson. It is safe to conclude from the analogy with the Maxwell field that the energy density is of the order of magnitude of $\operatorname{grad}^{2} \varphi \fallingdotseq g^{2} \lambda^{2}$ at the distance $1 / \lambda$. The volume of the region where the energy density has this value is of the order of magnitude of $1 / \lambda^{3}$; thus the total energy is $g^{2} / \lambda$. The energy of the meson is $\sim \mu c^{2}$. The fraction of time spent in the dissociated state is therefore

$$
\alpha=\frac{g^{2}}{\lambda \mu c^{2}}=\frac{g^{2}}{\hbar c}=\frac{1}{10} .
$$

The nuclear particles have so far been considered as point- or mono-poles, being the sources for the field around them. Accordingly, the field was spherically symmetrical. There is, however, evidence that these are not the only sources of the meson field. It is known that the proton neutron force depends essentially upon the relative spin directions of these two particles. The difference hetween the singlet and triplet potentials of the deuteron is of the same order of magnitude as the potential itself. It must therefore be concluded that the spin of a heavy particle acts also as a source for a meson field, the latter being no longer spherically symmetrical but having a signified direction in the direction of the spin. The dependence on angle of this field is that of a wave function of a particle in a $p$-state. Thus we attribute to the spin of a proton or neutron a 'mesonic dipole' $\sigma f / \lambda$ ( $\sigma$ is the spin of the heavy particle), where $f$ is another constant with the dimensions of a charge. At the distances of interest, $(1 / \lambda)$, this field has the same order of magnitude as the field of the mono-pole if the two constants $g$ and $f$ are of the same order of magni- tude. This assumption can account for the spin dependence of the nuclear forces.

If we look upon the dipole field from the particle aspect, we can describe it in the following way: The proton is also capable of emitting virtually a meson with angular momentum of one unit, the angular momentum having the same direction as the spin of the proton before the dissociation. In order to satisfy the conservation of angular momentum, the spin direction of the heavy particle in the dissociated state must be the opposite one. The fraction of time spent in this kind of dissociation is also of the order of magnitude $\alpha=f^{2} / \hbar c$ $=g^{2} / \hbar c$.

Considerations of this kind lead to the qualitative explanation of another very important feature, namely, the anomalous magnetic moments of the proton and neutron. It is clear that a meson with angular momentum 1 has a magnetic moment larger by a factor $M / \mu$ than $1 \mathrm{Bohr}$ nuclear magneton. This contributes to the magnetic moment of the proton an amount $\alpha M / \mu$ (in units of the nuclear magneton) which explains why the magnetic moments have such odd values. During the dissociation, the heavy particle is a neutron and has therefore no magnetic moment of its own. The heavy particle itself contributes therefore a magnetic moment $1-\alpha$. The total magnetic moment of the proton is therefore

$$
m_{P}=1-\alpha+\alpha M / \mu \text {. }
$$

Similarly, the magnetic moment of the neutron will be

$$
m_{N}=-\alpha-\alpha, M / \mu,
$$

where regard has been taken of the fact that the neutron emits a negative meson with the opposite sign of magnetic moment, and that in the dissociated state the neutron has become a proton with opposite spin direction. (In (7) and (8) no account has been taken of the fact that a proton can dissociate into states where the meson has no angular momentum. It can easily be seen that this does not alter the result appreciably.)

Since both $m_{P}$ and $m_{N}$ are of the order of magnitude unity, we see that $\alpha$ must be of the order of magnitude of $\mu / M$. But this is just the relation (4) derived from the binding energy and size of the deuteron, and gives further support for the assumption that this relation is not accidental. If we insert in (7) and (8) the observed values of $m_{P}=2.78$ and $m_{N}=-1.93$, we have two equations for a new independent determination of $\alpha$ and $M / \mu$. We find

$$
\alpha=0.075 \text { and } M / \mu=20 \text {, }
$$

in reasonable agreement with the values previously obtained for these universal constants.

The consistency of these results suggests that the 
fundamental concepts of this theory are correct. We must not forget, however, that we have restricted our considerations to distances of the order of magnitude $1 / \lambda$. Little can be said at present about the contributions of smaller distances. All we can say is that their contributions to the effects considered are at most of the same order of magnitude as those from greater distances. Our results are largely independent of the particular formalism chosen to describe the meson field, so long as it conforms with what we had to postulate. Many important details, however, do depend on the value chosen for the spin of the meson ( 0 or 1 , or both); for example the order of the levels in the deuteron, and the behaviour of the meson during collisions with other particles and light quanta. In some cases more or less serious difficulties arise if the theory is applied to distances smaller than $1 / \wedge$. In this respect no definite decision has been reached yet, and we must expect further information from future developments.
Finally, we must also mention the $\beta$-decay of the meson. This was originally a second idea of Yukawa's, quite independent of his theory of the nucleus. It was assumed that the meson can decay directly into an electron and a neutrino. The decay of the meson has since been confrmed experimentally. The great advantage of this hypothesis is that it reduces the $\beta$-decay to a much simpler form. Whereas a nucleus decays emitting the electron into a continuous range of energy, the electron emitted by a free meson can only have the energy $\mu c^{2} / 2$ (leaving the other half for the neutrino). The $\beta$-decay of a nucleus then takes place by means of an intermediate emission of a meson but the fundamental process underlying the $\beta$-decay of a nucleus is the decay of the meson. It is clear that a detailed study of the meson decay will give us very valuable information about the most important problem of the existence of the neatrino. A detailed discussion, however, lies outside the scope of this article.

\section{BIOLOGICAL ACTION OF VITAMINS By Dr. ElizABETH M. KoCH \\ UnIversity of Chicago}

SOME of the outstanding developments in vita$S_{\text {min }}$ research reported in the symposium on the "Biological Action of Vitamins", held at the University of Chicago as part of its fiftieth anniversary celebration during September 15-17, were :

(1) The further clarification of the functions of diphosphothiamin in carbohydrate metabolism by Mr. Severo Achoa, of Washington University, in St. Louis ; (2) The separation of a new B vitamin, folic acid, a yeast growth stimulant announced by Mr. Roger J. Williams, of the University of Texas ; (3) The proof of the identity of biotin, vitamin $\mathrm{H}$, and co-enzyme $\mathrm{R}$, presented by $\mathrm{Mr}$. Vincent du Vigneaud of Cornell Medical College, New York City ; (4) The isolation from egg white of avidin, a substance which combines with and thus inactivates biotin, also reported by $\mathrm{Mr}$. Roger J. Williams; (5) The description of a new choline deficiency effect in young rats characterized by hæmorrhagic degeneration of the kidneys, described by Mr. Wendell H. Griffith, of St. Louis University, in St. Louis ; (6) The description of the symptoms of human ariboflavinosis by Dr. W. H. Sebrell, of the United States Public Health Service ; (7) The extension of vitamin therapy in human nutritional diseases, reported by Dr. Norman Joliffe, of New York University, by Dr. David L. Smith, of Duke University, and by
Dr. Tom Spies, of the University of Cincinnati ; (8) The use of vitamin $K$ to prevent bleeding in patients having a low prothrombin content in the blood, reported by Drs. Harry P. Smith and Emory D. Warner, of the University of Iowa.

$\mathrm{Mr}$. Achoa, in his discussion of the functions of diphosphothiamin, pointed out that through its effect on the oxidation of pyruvic acid, diphosphothiamin may be expected to influence various phases of carbohydrate metabolism. This is true because of the fact that pyruvate oxidation causes the storage of a considerable amount of energy in the form of adenosine triphosphate. We may expect diphosphothiamin to be indirectly involved in the synthesis of glycogen from glucose and in the conversion of fructose to glucose, since these reactions are driven by the mobilization of the phosphate bond energy in adenosine triphosphate.

Mr. Achoa also suggested the possibility that diphosphothiamin may aid in the absorption of sugar from the intestines and in resorbing glucose in the kidney tubules. It also may be involved in the process of forming carbohydrate molecules from lactic acid or from pyruvic acid. This vitamin complex likewise hastens production by the body of acetylcholine, an agent involved in the neuromuscular mechanism.

Folic acid, so named because it is most abundant 